\title{
Cardiopatias na gestação: aspectos clínicos
}

\author{
Heart diseases in pregnancy: clinical aspects \\ Cardiopatías en el embarazo: aspectos clínicos
}

Priscylla Frazão Rodrigues ${ }^{1 *}$, Adrielly Cristhine Gonçalves Araujo ${ }^{1}$, Andressa Carvalho Pereira ${ }^{1}$, Brenda Larissa Andrade Viana ${ }^{1}$, Igor dos Santos Cavalcante ${ }^{1}$, Isabella Pires Gomes Mendes ${ }^{1}$, Juliano Luiz de Souza ${ }^{1}$, Érica de Araújo Silva Mendes ${ }^{1}$, Nayana Alves de Brito Melo Okasaki ${ }^{1}$, Elias de Carvalho Magalhães Neto'.

\section{RESUMO}

Objetivo: Analisar as principais cardiopatias na gravidez, enfatizando suas interferências e associações com complicações e desfechos no ciclo gravídico-puerperal. Métodos: Trata-se de um estudo de revisão literária integrativana qual a coleta de dados foi realizada nas bases de dados Scientific Eletronic Library Online (SciELO), Literatura LatinoAmericana e do Caribe (LILACS) e PubMed, utilizando como descritores "Cardiopatias", "Gravidez" e "Gravidez de alto risco". Os dados foram analisados e discutidos com a literatura atual. Resultados: A partir da análise dos artigos, foram identificadas entre as principais alterações, as cardiopatias reumáticas e congênitas. As principais complicações foram a insuficiência cardíaca, as arritmias e os eventos tromboembólicos. As síndromes hipertensivas e os quadros hemorrágicos estiveram associados ao aumento da morbimortalidade nas gestantes. Diante desses agravos, foram pontuados alguns aspectos do manejo clínico em gestantes. Considerações finais: Conclui-se que as cardiopatias correspondem a uma importante causa de morbimortalidade durante a gravidez e aumentam os riscos de complicações obstétricas. Assim, é importante um atendimento individualizado, precoce e multidisciplinar dessas gestantes, para minimizar as complicações materno-fetais.

Palavras-Chave: Cardiopatias, Gravidez, Gravidez de alto risco.

\begin{abstract}
Objective: To analyze the main heart diseases in pregnancy, emphasizing their interference and associations with complications and outcomes in the pregnancy-puerperal cycle. Methods: This is an integrative literary review study in which data collection was carried out in the databases of the Online Electronic Scientific Library (SciELO), Latin American and Caribbean Literature (LILACS) and PubMed, using "Heart Diseases" as descriptors, "Pregnancy" and "High-risk pregnancy". The data were analyzed and discussed with the current literature. Results: From the analysis of the articles, rheumatic and congenital heart diseases were identified among the main alterations. The most frequent complications were heart failure, arrhythmias and thromboembolic events. Hypertensive syndromes and hemorrhagic conditions were associated with increased morbidity and mortality in pregnant women. In view of these problems, some aspects of clinical management in pregnant women were scored. Final considerations: It is concluded that the heart diseases are an important cause of morbidity and mortality during pregnancy and increase the risk of obstetric complications. Thus, it is important to provide individualized, early and multidisciplinary care to these pregnant women, to minimize maternal-fetal complications.
\end{abstract}

Keywords: Heart diseases, Pregnancy, High-risk pregnancy.

\section{RESUMEN}

Objetivo: Analizar las principales enfermedades cardíacas en el embarazo, enfatizando su interferencia y asociaciones con complicaciones y resultados durante el embarazo y el posparto. Métodos: Este es un estudio integrador de revisión literaria en el cual la recolección de datos se realizó en las bases de datos de la Biblioteca Científica Electrónica en Línea (SciELO), Literatura Latinoamericana y del Caribe (LILACS) y PubMed, utilizando "Enfermedades del Corazón" como descriptores, "Embarazo" y "Embarazo de alto riesgo". Los datos fueron analizados y discutidos con la literatura actual. Resultados: A partir del análisis de los artículos, se identificaron entre las principales alteraciones las enfermedades cardíacas reumáticas y congénitas. Las principales complicaciones fueron insuficiencia cardíaca, arritmias y eventos tromboembólicos. Los síndromes hipertensivos y las condiciones hemorrágicas se asociaron con un aumento de la morbilidad y la mortalidad en mujeres embarazadas. En vista de estos problemas, se puntuaron algunos aspectos del manejo clínico en mujeres embarazadas. Consideraciones finales: Se concluye que las cardiopatías son una causa importante de morbilidad y mortalidad durante el embarazo y aumentan el riesgo de complicaciones obstétricas. Por lo tanto, es importante proporcionar atención individualizada, temprana y multidisciplinaria a estas mujeres embarazadas, para minimizar las complicaciones materno-fetales.

Palabras clave: Cardiopatías, Embarazo, Embarazo de alto riesgo.

1Universidade Federal do Delta do Parnaíba (UFDPar), Parnaíba - PI. *E-mail: priscyllafrazao1995@gmail.com 


\section{INTRODUÇÃO}

A cardiopatia é considerada a maior causa de morte materna indireta durante o ciclo gravídico-puerperal (TEDOLDI CL, et al., 2009). O Brasil, por exemplo, supera as estatísticas internacionais de incidência de cardiopatia na gestação, chegando a um índice de 4,2\% (MENEGUIN S, et al., 2016). Estima-se que, em países industrializados, de 0,2 a 4\% das gestações sejam complicadas por doenças cardíacas (LAGE EM e BARBOSA AS, 2012).

Sendo assim, o manejo clínico das doenças cardíacas, associado ao conhecimento dos riscos relativos a essas patologias, o seu diagnóstico precoce e o delineamento de intervenções em todo o ciclo gravídico puerperal são de extrema importância para minimizar as complicações materno-fetais e potencializar a qualidade de vida dessas mulheres (AVILA WS, et al., 2020; AMORIM TV, et al, 2017).

A gestação representa um momento de grandes mudanças fisiológicas, incluindo alterações cardiovasculares, que são responsáveis por assegurar o desenvolvimento fetal, garantindo uma perfusão placentária adequada, e por preparar a mãe para o parto.

Apesar de importantes nesse período, os ajustes cardiovasculares podem não ser suportados por mulheres sem reserva cardíaca normal (DE MELO MOREIRA V, 2019). Dentre essas alterações estão o aumento da frequência cardíaca (de 15 a 30\%), a elevação do débito cardíaco (de 30 a 50\%) e o aumento do volume sanguíneo (de 40 a $50 \%$ ).

Ocorre também a redução da pressão arterial e a diminuição substancial da resistência vascular periférica, uma vez que há aumento na produção endotelial de prostaciclina e óxido nítrico (LAGE EM e BARBOSA AS, 2012; DE MELO MOREIRA V, 2019).

Tais alterações tem início no primeiro trimestre e são máximas quando a gestante atinge a 24a ${ }^{\mathrm{a}}$ semana, com aumento no pós-parto (MENEGUIN S, et al., 2016). Sabe-se que essa sobrecarga hemodinâmica pode gerar descompensação clínica de doenças pré-existentes ou revelar patologias cardíacas até então desconhecidas (AVILA WS, et al., 2020; RAMLAKHAN KP, et al., 2020).

As principais etiologias da cardiopatia durante a gravidez são as lesões congênitas e reumáticas. Definese como cardiopatia congênita o conjunto de alterações estruturais ou funcionais já presentes ao nascimento, ainda que diagnosticadas posteriormente.

Tais alterações podem ou não causar cianose, sendo assim classificadas em cianóticas e acianóticas. Entre as cardiopatias congênitas cianóticas, a Tetralogia de Fallot é a mais frequente. Já entre as cardiopatias congênitas acianóticas destacam-se a comunicação interatrial e interventricular e a persistência do canal arterial. A lesão cardíaca congênita é considerada a segunda maior causa indireta de mortalidade materna, alcançando até $20 \%$ dos óbitos por doença cardíaca (AVILA WS, et al., 2020).

A cardiopatia reumática corresponde a uma sequela da febre reumática aguda, doença inflamatória que ocorre após um episódio de faringoamigdalite bacteriana, tratada de forma inadequada. Essas alterações decorrem da resposta imune tardia a esta infecção em populações geneticamente susceptíveis.

A febre reumática afeta principalmente crianças e adultos jovens e é frequentemente associada à pobreza e às más condições de vida (BARBOSA PJB, et al., 2009). A febre reumática aguda é rara durante a gravidez, mas seu diagnóstico deve ser considerado em gestantes adolescentes sem profilaxia prévia que apresentem quadro de insuficiência cardíaca grave (AVILA WS, et al., 2020).

No Brasil, a doença reumática é a principal causa de cardiopatia durante a gestação, correspondendo a cerca de $50 \%$ do total. Já em países desenvolvidos, as cardiopatias congênitas são predominantes, devido à redução e ao tratamento precoce da febre reumática nesses locais, bem como ao aumento da sobrevida das mulheres com cardiopatias congênitas (LAGE EM e BARBOSA AS, 2012).

As condições socioeconômicas precárias representam as principais dificuldades enfrentadas pelas autoridades de saúde na luta pela erradicação da febre reumática (GALVÃO ALC e GREZZANA GB, 2005). Segundo Avila WS, et al. (2020), a insuficiência cardíaca (IC), os distúrbios tromboembólicos e as arritmias são complicações comuns associadas à mortalidade materna em mulheres cardiopatas. 
Muitas patologias cardíacas podem resultar em insuficiência cardíaca durante a gravidez. De acordo com a Sociedade Brasileira de Cardiologia, a insuficiência cardíaca é uma condição clínica na qual o coração não consegue bombear o sangue de maneira a atender às necessidades metabólicas do organismo, ou, só consegue fazê-lo, através de um esforço maior que o usual, elevando as pressões de enchimento (ROHDE LE, et al., 2018).

As alterações hormonais e hemodinâmicas da gravidez favorecem o surgimento de arritmias cardíacas. Segundo a Sociedade Brasileira de Arritmias Cardíacas, a arritmiaé uma alteração que ocorre na formação ou na condução do estímulo elétrico do coração, podendo provocar modificações do ritmo cardíaco. A maioria é benigna, mas em $6 \%$ dos casos pode levar a complicações e aumentar as chances de efeitos adversos para a mãe e o feto (FELCZAK C, et al., 2018).

As arritmias mais frequentes na gestação são a fibrilação atrial e a taquicardia supraventricular, sendo a última a arritmia sintomática mais comum e mais comumente observada no final da gestação (RAMLAKHAN $\mathrm{KP}$, et al., 2020).

Os distúrbios sustentados do ritmo cardíaco podem comprometer o desenvolvimento fetal, determinando baixo peso, parto prematuro e mais indicações de cesárea (AVILA WS, et al., 2020). Recomenda-se que gestantes com cardiopatia congênita associada a arritmia e insuficiência cardíaca sejam monitorizadas cuidadosamente, devido ao risco aumentado de comprometimento hemodinâmico materno e fetal (LAGE EM e BARBOSA AS, 2012).

Outra alteração importante é a síndrome hipertensiva durante a gestação. Corresponde à complicação médica mais comum na gravidez, afetando de 5 a $10 \%$ das gestações em todo o mundo. Define-se como hipertensão gestacional uma elevação pressórica "nova" que surge após a $20^{\underline{a}}$ semana de gestação, na ausência de proteinúria, anormalidades bioquímicas ou hematológicas.

Cerca de um quarto das mulheres com hipertensão gestacional pode evoluir para pré-eclâmpsia com prognóstico desfavorável. Define-se como pré-eclâmpsia grave a hipertensão de início agudo com pressão sistólica igual ou superior a $160 \mathrm{mmHg}$ e pressão diastólica igual ou superior a $110 \mathrm{mmHg}$ (AVILA WS, et al., 2020).

Estima-se que os distúrbios hipertensivos sejam responsáveis por 10-16\% da mortalidade materna em todo o mundo (RAMLAKHAN KP, et al., 2020). Todo esse contexto revela a importância do atendimento individualizado e multidisciplinar dessas gestantes. $O$ aconselhamento pré-concepcional também é um processo necessário e deve incluir a discussão sobre o risco para a mãe e para o feto e a chance de hereditariedade da cardiopatia (LAGE EM e BARBOSA AS, 2012).

Assim, o presente estudo teve como objetivo analisar através de uma revisão integrativa as principais cardiopatias na gravidez, enfatizando suas interferências e associações com complicações e desfechos no ciclo gravídico-puerperal.

\section{MÉTODOS}

Trata-se de uma revisão de literatura integrativa, obedecendo as normas pertinentes de levantamento, seleção, ordenação e síntese de material publicado por outros autores. Para este fim, foi determinada a seleção do tema e definição do objetivo de pesquisa.

Posteriormente, foi feita a determinação dos fatores de inclusão e exclusão para a seleção dos artigos, seguida de análise e interpretação dos resultados para a síntese da revisão. Dessa forma, o tema definido foi "cardiopatias em gestantes" e objetivou-se analisar seus diversos aspectos clínicos, visando-se compreender, não apenas como as afecções que envolvem o coração afetam o desenvolvimento da gestação, mas também, como ocorre o manejo e o acesso dessas pacientes no serviço de saúde.

Foi realizada uma pesquisa bibliográfica nas bases de dados: Scientific Eletronic Library Online (SciELO), Literatura Latino-Americana e do Caribe (LILACS) e PubMed utilizando os descritores: "Cardiopatias", "Gravidez" e "Gravidez de alto risco". 
Para aprimoramento da busca, foi utilizado o operador booleano "AND" e artigos de relevância ao tema foram selecionados. Na base SciELO, ao utilizar os três descritores associados encontrou-se quatro artigos. A seguir os descritores foram utilizados em pares: usando "cardiopatias" e "gravidez" encontrou-se dezoito artigos, com "cardiopatias" e "gravidez de alto risco" não houve resultado e com "gravidez" e "gravidez de alto risco" encontrou-se duzentos e vinte e três resultados.

Na LILACS, a composição dos três descritores obteve dez artigos, usando o par "cardiopatias" e "gravidez" obteve-se cento e dezessete artigos, com o par "cardiopatias" e "gravidez de alto risco" obteve-se dezenove resultados e com o par "gestação" e "gestação de alto risco" obteve-se sessenta e sete trabalhos.

Ao utilizar os três descritores na base PubMed, nenhum artigo foi encontrado. Com a associação "cardiopatia" e "gravidez" foi encontrado um artigo, como o par "cardiopatia" e "gravidez de alto risco" não houve resultado e no par "gravidez" e "gravidez de alto risco" foram encontrados três artigos.

Os critérios de inclusão foram: artigos científicos disponibilizados de forma gratuita e integral, que fossem pesquisas em seres humanos, publicados entre 2000 e 2020 e tratassem de problemas cardíacos em gestantes. Quanto aos critérios de exclusão, foram desconsiderados materiais que não fossem artigos científicos, não estivessem dentro do período de 2000 a 2020 e estudos que não tivessem de acordo com o tema selecionado. (Figura 1)

Figura 1 - Fluxograma detalhando cada etapa do processo de seleção de artigos para a revisão.

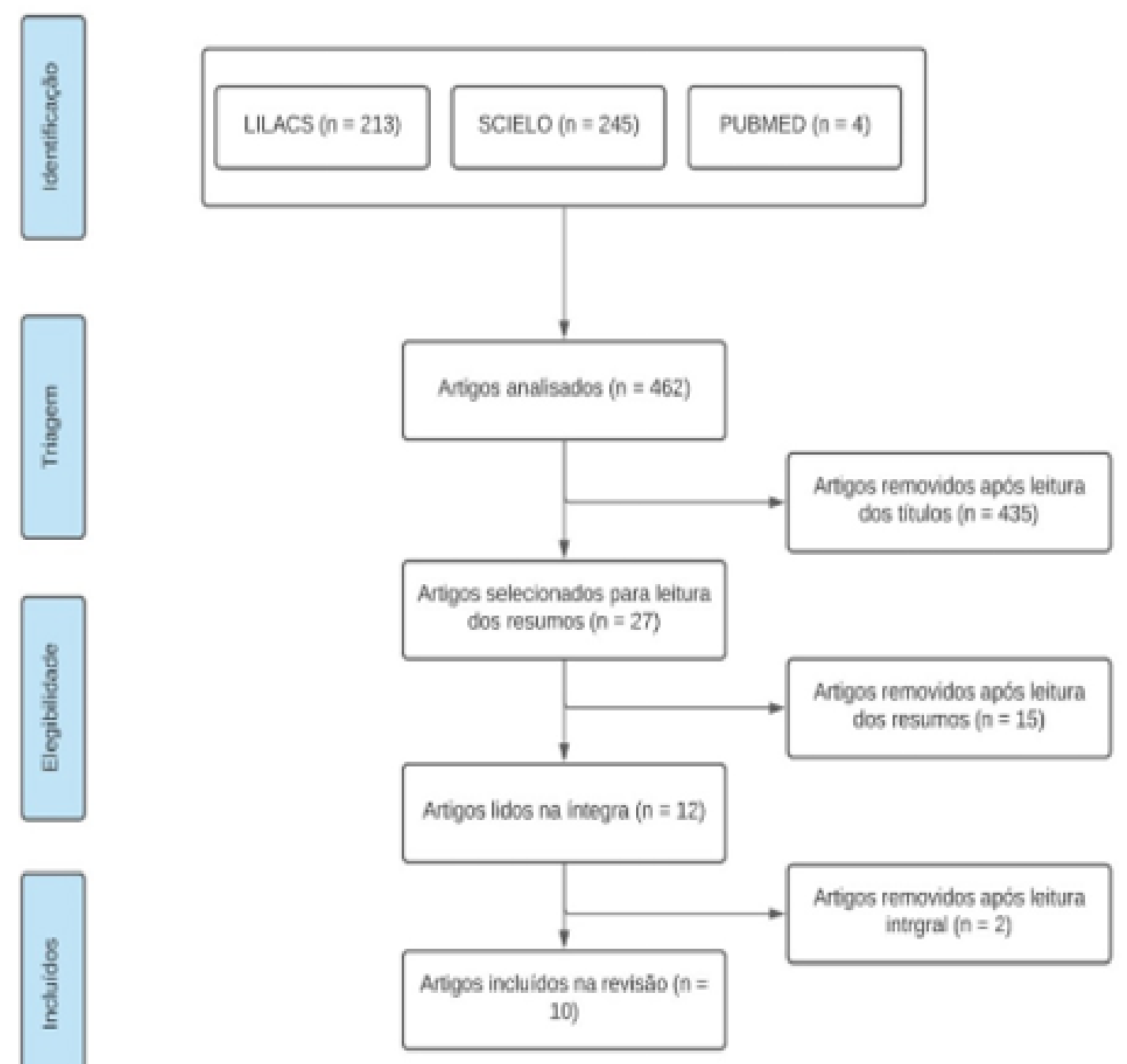

Fonte: Rodrigues PF, et al., 2020. 
A seleção dos estudos foi realizada de forma independente por dois pesquisadores a partir da análise dos títulos, resumos e textos completos das publicações. Assim, quatrocentos e trinta e cinco (435) artigos cujos títulos fogem do tema de interesse foram descartados, bem como quinze (15) que, após leitura de resumo, não se enquadraram nos fatores de inclusão. Pela leitura integral dos textos, dois (2) artigos foram excluídos (Quadro 1).

Quadro 1 - Artigos selecionados para a composição do estudo, abordando a revista, ano de publicação, objetivos e seus respectivos resultados principais.

\begin{tabular}{|c|c|c|c|}
\hline Obra & Ano & Periódico & Objetivo \\
\hline $\begin{array}{l}\text { Anticoagulação, gravidez e } \\
\text { cardiopatia. Uma tríade, três } \\
\text { domínios e cinco momentos }\end{array}$ & 2005 & $\begin{array}{l}\text { Arquivos } \\
\text { brasileiros de } \\
\text { cardiologia }\end{array}$ & $\begin{array}{l}\text { Discutir o uso de anticoagulação em } \\
\text { gestantes cardiopatas, a partir de } \\
\text { estudos do setor de cardiopatia e } \\
\text { gravidez da unidade clínica de } \\
\text { valvopatias do incor. }\end{array}$ \\
\hline $\begin{array}{l}\text { Anticoagulação durante gravidez de } \\
\text { mulher portadora de cardiopatia }\end{array}$ & 2010 & $\begin{array}{l}\text { Jornal vascular } \\
\text { brasileiro }\end{array}$ & $\begin{array}{l}\text { Discutir o risco materno ao } \\
\text { tromboembolismo e as propriedades } \\
\text { dos anticoagulantes indicados para a } \\
\text { sua prevenção e o tratamento de } \\
\text { mulher portadora de cardiopatia }\end{array}$ \\
\hline $\begin{array}{l}\text { Complicações maternas associadas à } \\
\text { via de parto em gestantes } \\
\text { cardiopatas em um hospital terciário } \\
\text { de fortaleza, ce }\end{array}$ & 2012 & $\begin{array}{l}\text { Revista brasileira } \\
\text { de ginecologia e } \\
\text { obstetrícia }\end{array}$ & $\begin{array}{l}\text { Observar a associação entre } \\
\text { complicações maternas e o tipo de } \\
\text { parto em gestantes cardiopatas e } \\
\text { identificar os possíveis fatores clínicos } \\
\text { e obstétricos, implicados na } \\
\text { determinação da via de parto }\end{array}$ \\
\hline Cardiopatias e gravidez & 2012 & Revista femina & $\begin{array}{l}\text { Revisão de literatura sobre cardiopatias } \\
\text { na gravidez, com enfoque no } \\
\text { aconselhamento pré-concepcional e o } \\
\text { manejo pré-natal. }\end{array}$ \\
\hline $\begin{array}{l}\text { Consideraciones generales de la } \\
\text { embarazada con enfermedad } \\
\text { cardiaca congénita y adquirida }\end{array}$ & 2013 & $\begin{array}{l}\text { Revista chilena } \\
\text { de anestesia }\end{array}$ & $\begin{array}{l}\text { Analisar o perfil epidemiológico e } \\
\text { condições clínicas de gestantes com } \\
\text { cardiopatia congênita ou adquirida }\end{array}$ \\
\hline $\begin{array}{l}\text { Fatores associados à morte materna } \\
\text { em unidade de terapia intensiva }\end{array}$ & 2016 & $\begin{array}{l}\text { Revista brasileira } \\
\text { de terapia } \\
\text { intensiva }\end{array}$ & $\begin{array}{l}\text { Identificar os fatores associados à } \\
\text { morte materna em pacientes internadas } \\
\text { em unidade de terapia intensiva. }\end{array}$ \\
\hline $\begin{array}{c}\text { Promoção da saúde materna a partir } \\
\text { do vivido do parto de mulheres } \\
\text { cardiopatas }{ }^{\star}\end{array}$ & 2017 & $\begin{array}{l}\text { Revista cogitare } \\
\text { enfermagem }\end{array}$ & $\begin{array}{l}\text { Analisar os significados da via de parto } \\
\text { a partir da vivência do risco gestacional } \\
\text { da mulher portadora de cardiopatia }\end{array}$ \\
\hline $\begin{array}{c}\text { Profile of cardiac pregnant women: } \\
\text { high-risk }\end{array}$ & 2018 & $\begin{array}{l}\text { Revista cogitare } \\
\text { enfermagem }\end{array}$ & $\begin{array}{c}\text { Caracterizar gestantes com cardiopatia } \\
\text { gestacional de alto risco atendidas no } \\
\text { setor secundário da } 3^{\underline{a}} \text { região de saúde } \\
\text { do paraná }\end{array}$ \\
\hline $\begin{array}{l}\text { Gravidez em portadoras de } \\
\text { cardiopatias congênitas complexas: } \\
\text { um constante desafio }\end{array}$ & 2019 & $\begin{array}{l}\text { Arquivos } \\
\text { brasileiros de } \\
\text { cardiologia }\end{array}$ & $\begin{array}{l}\text { estudar a evolução materno-fetal das } \\
\text { gestantes portadoras de cardiopatias } \\
\text { congênitas complexas e analisar as } \\
\text { variáveis presumíveis de prognóstico }\end{array}$ \\
\hline $\begin{array}{l}\text { Heart disease and pregnancy: state of } \\
\text { the art }\end{array}$ & 2019 & $\begin{array}{l}\text { Revista } \\
\text { portuguesa de } \\
\text { cardiologia }\end{array}$ & $\begin{array}{l}\text { Organizar como ocorre a abordagem } \\
\text { de gestantes com cardiopatias de alto } \\
\text { risco }\end{array}$ \\
\hline
\end{tabular}

Fonte: Rodrigues PF, et al., 2020. 


\section{REVISÃO BIBLIOGRÁFICA}

O ciclo gravídico provoca alterações cardiovasculares fisiológicas no corpo da mulher para tornar possível o processo de desenvolvimento fetal e do parto (LAGE EM e BARBOSA AS, 2012). Essas mudanças acentuam riscos e devem ser monitorizados frequentemente, principalmente quando relacionados a cardiopatias pré-existentes, sendo este um importante fator de morbimortalidade, não apenas no período gestacional, mas também, no puerpério (FELCZAK C, et al., 2018).

Em Avila WS, et al. (2019), um estudo observacional, retrospectivo, realizado em um Centro de Cardiologia e Obstetrícia no período de 10 anos, verificou-se que em 435 gestantes com cardiopatia congênita (CC), 40 possuíam cardiopatia congênita complexa (CCC). Ocorreram complicações em $59,5 \%$ dos casos, sendo $4,7 \%$ de mortes maternas associadas a hipoxemia; $40,5 \%$ de bebês prematuros; $16,6 \%$ de mortes fetais e 30,9\% de complicações maternas, com prevalência de insuficiência cardíaca (IC) e arritmias.

Segundo Lage EM e Barbosa AS (2012), as cardiopatias congênitas e reumáticas se destacam como etiologia dessas complicações. Isso se relaciona ao fato de alterações estruturais complexas, existentes nessas doenças, estarem associadas a maiores chances de desenvolvimento destes agravos, destacandose ainda a maior ocorrência de eventos tromboembólicos (DRENTHEN W, et al., 2007).

Cavalcante MS, et al. (2012) analisaram prontuários obstétricos de cardiopatas de um hospital de Fortaleza, Ceará, internadas de 2006 a 2007, nas quais a comunicação interatrial apareceu como a CC mais frequente, observando-se ainda a estenose mitral como a de maior incidência entre as adquiridas. Esses dados foram concordantes com o encontrado a nível nacional, em que $55 \%$ das cardiopatias na gravidez são de origem reumática sendo 70 a $80 \%$ casos de estenose mitral (MOREIRA WR e ANDRADE LC, 2009). Em relação às cardiopatias congênitas, as acianóticas são as mais comuns, sendo mais frequente a comunicação interatrial (CIA) e a comunicação interventricular (CIV), corroborando com os dados apresentados (BORN D, 2009).

Conforme Guimarães T, et al. (2019), a IC é a complicação mais frequente das cardiopatias congênitas cianóticas, representando 32\% das gestações. No estudo de Lage EM e Barbosa AS (2012) verificou-se, em portadoras da síndrome de Eisenmenger, que a taxa de mortalidade materna é de $40 \%$ e é superior em gestantes submetidas a cesarianas (75\%). A tetralogia de Fallot também eleva o risco de IC, uma vez que há a presença de disfunção ventricular direita como consequência da regurgitação pulmonar grave, disfunção do ventrículo esquerdo ou de hipertensão pulmonar (LAGE EM e BARBOSA AS, 2012).

Ademais, a IC pode resultar de diferentes condições clínicas, dentre elas, têm-se a fibrilação atrial e patologias causadoras de comunicação interventricular, especialmente em casos de defeito septal grande. Em casos de gestantes com estenose aórtica grave, o aumento do débito cardíaco é restrito e isso resulta em aumento da pressão sistólica do ventrículo esquerdo, que origina ou intensifica a IC (LAGE EM e BARBOSA AS, 2012).

Nesse sentido, observa-se que a sobrevivência fetal está associada à saturação de oxigênio materna em repouso: uma saturação $\leq 85 \%$ corresponde a $12 \%$ de sobrevida fetal (GUIMARÃES T, et al., 2019). Tornase, então, necessário que a equipe de atendimento da gestante cardiopata esteja preparada para prevenção, monitoramento e tratamento das principais complicações, como a IC, no intraparto e puerpério, em virtude do elevado risco materno e fetal nestas pacientes (AVILA WS, et al., 2020).

As arritmias cardíacas são outras complicações frequentes em adultos acometidos por CC, de acordo com o estudo quantitativo, realizado em 2016, a partir da análise de sessenta prontuários de gestantes cardíacas da Clínica de Gravidez de Alto Risco de Campos Gerais, Paraná, em que se percebeu que as arritmias aumentam as taxas de cesariana, representando $85,7 \%$ dos casos (FELCZAK C, et al., 2018).

No entanto, segundo a Sociedade Brasileira de Cardiologia (SBC), para mulheres grávidas com cardiopatias, a maioria das arritmias são benignas, apenas $6 \%$ levam a complicações, e quando estas resultam em internação, e ocorre indicação de interrupção da gestação, é que a recomendação de cesárea diminui a morbimortalidade perinatal nestas gestantes (SAINTRAIN SV, et al., 2016). 
Outra etiologia relacionada a essas complicações é a doença reumática, cuja manutenção da sua profilaxia durante a gravidez é essencial,segundo a Sociedade Brasileira de Cardiologia, sendo a penicilina benzatina a droga de escolha (AVILA WS, et al., 2020). Esse agravo corresponde a 50\% dos casos de cardiopatias em gestantes no Brasil,o qual necessita de substituição valvar por próteses, uma vez que a doença valvar reumática é um fator de risco para tromboembolismo na gestação (AVILA WS, et al., 2019; FELCZAK C, et al., 2018).

Conforme a SBC, as próteses, tanto as biológicas (PB) quanto as mecânicas (PM), melhoram a capacidade funcional da perspectiva hemodinâmica durante a gravidez. Apesar de a PB ser a substituta que apresenta pontos favoráveis, uma vez que não requer anticoagulação como as $\mathrm{PM}$, ainda não há prótese valvar segura, necessitando de avaliação acerca dos riscos e benefícios (AVILA WS, et al., 2020).

Ao avaliar a utilização de anticoagulantes, concomitantemente à análise da substituição valvar, em gestantes cardiopatas, as diretrizes orientam a avaliação entre os prejuízos e as vantagens, tanto maternos quanto fetais. É preferencial a utilização da heparina de baixo peso molecular (HBPM) no manejo clínico dessas pacientes, visto que não atravessa a barreira placentária e possuem meia-vida curta, permitindo uma anestesia regional de emergência (AVILA WS, 2010; GOMEZ R e NAZAR C, 2013).

No entanto, não se pode negar que o uso prolongado pode gerar trombocitopenia, osteoporose e quadros hemorrágicos, e, devido a isso, em casos de pacientes portadoras de próteses mecânica com anticoagulação permanente, ainda se utiliza varfarina no segundo trimestre (AVILA WS, et al., 2019; AVILA WS e GRINBERG M, 2005), sendo, pois, essencial a avaliação de cada caso.

Ainda, conforme o estudo qualitativo de Amorim TV, et al. (2017), observou-se que outros fatores, diferentes das $\mathrm{CC}$, cardiopatias adquiridas, síndromes hipertensivas e quadros hemorrágicos, também podem contribuir para a elevação da morbimortalidade nas gestantes. Entre esses fatores, nota-se os de causas subjetivas, como a ansiedade, uma vez que ela pode imprimir aumento de até $60 \%$ do débito cardíaco, demonstrando a importância de um manejo clínico amplo, que contemple todos os aspectos do ciclo gravídico.

Assim, diante das complicações apresentadas pelas gestantes cardiopatas, observa-se que, segundo o Ministério da Saúde, é necessário um pré-natal precoce e um acompanhamento multidisciplinar dessas pacientes. Em avaliação pré-concepcional, deve-se analisar os potenciais riscos materno-fetais expondo as alternativas terapêuticas, como o tratamento para algumas doenças cardíacas e analisar as possibilidades de reparação cirúrgica, além do desaconselhando da gravidez em alguns casos, assim como preconiza a Organização Mundial de Saúde (OMS) (AVILA WS, et al., 2019; GOMEZ R e NAZAR C, 2013).

No estudo observacional de Avila WS, et al. (2019), já citado, ocorreram 7 perdas fetais (16,6\%), 2 recémnascidos $(4,7 \%)$ com cardiopatia congênita e 17 bebês prematuros (40,5\%). Sabe-se que o melhor prognóstico materno-fetal decorre de alguns aspectos do manejo clínico de gestantes com cardiopatias, como o planejamento da gravidez associado a estratificação dos riscos, assim como o aconselhamento no período pré-concepcional. Para que isso aconteça, torna-se necessária a realização de um exame clínico cauteloso aliado a resultados de radiografias de tórax, eletrocardiograma, oximetria percutânea, ecocardiograma e cateterismo cardíaco (LAGE EM e BARBOSA AS, 2012).

Além disso, as primeiras 24 horas do período pós-parto configuram como as de maior importância da monitoração no puerpério, devido ao maior risco de complicações cardiovasculares, destacando-se quadros característicos de IC e balanço hídrico (GÓMEZ R e NAZAR C, 2013). Em UTI, as gestantes cardiopatas de alto risco devem ser monitoradas por 24 a 48 horas, uma vez que esse período corresponde a instabilidades hemodinâmicas e elevado risco de óbito. Destaca-se, também, que monitoramento e manejo volêmico inapropriados dão margem a disfunções cardiovasculares (AVILA WS, et al., 2020).

Apesar das diversas complicações enfrentadas pelas pacientes cardiopatas, a pré-existência de cardiopatias não impede a ocorrência de gestações. Dessa forma, é importante salientar os fatores sociais que estão diretamente relacionados com o planejamento familiar. Nessa perspectiva, o baixo nível de escolaridade de gestantes com cardiopatias é um fator sugestivo para o desconhecimento dos riscos que problemas cardíacos trazem durante a gravidez. Isso ocorre, pois o limitado acesso à informação dificulta a compreensão do processo de adoecimento e do acesso a serviços de saúde (FELCZAK C, et al., 2018).

REAS/EJCH | Vol.12(11) | e4987 | DOI: https://doi.org/10.25248/reas.e4987.2020 Página 7 de 8 
Segundo pesquisa realizada por Cavalcante MS, et al. 2012, em um hospital de referência no Ceará, a idade média das gestantes com cardiopatia foi de 26,4 anos e cerca de $40 \%$ possuíam até 7 anos de estudos, com quase $10 \%$ possuindo menos de 3 anos de escolaridade. Esse é um cenário de contínuo crescimento, uma vez que falta a orientação prévia e ocorre dificuldade de acesso aos serviços de atendimento pré-natal (AVILA WS, et al., 2019; CAVALCANTE MS, et al., 2012).

\section{CONSIDERAÇÕES FINAIS}

As principais etiologias da cardiopatia durante a gravidez são as lesões congênitas e reumáticas, sendo a doença reumática a principal causa de cardiopatia durante a gestação no Brasil. Outras complicações comuns associadas à mortalidade materna em cardiopatas são: insuficiência cardíaca, os distúrbios tromboembólicos e as arritmias. Entretanto, a pré-existência de cardiopatias não impede a ocorrência de gestações e, assim, é imprescindível o atendimento individualizado e multidisciplinar dessas gestantes. Desse modo, para atenuar os riscos e complicações de cardiopatias na gestação, deve ser feito uma avaliação pré-concepcional, analisando potenciais riscos materno-fetais e expondo as alternativas terapêuticas, além de um pré-natal precoce, com acompanhamento e aconselhamento materno.

\section{REFERÊNCIAS}

1. AMORIM TV, et al. Promoção da saúde materna a partir do vivido do parto de mulheres cardiopatas. Cogitare Enferm, 2017; (22)4: e51641.

2. ÁVILA WS. Anticoagulação durante gravidez de mulher portadora de cardiopatia. J Vasc Bras, 2010; 9(1): 42-45.

3. AVILA WS, GRINBERG M. Anticoagulação, Gravidez e Cardiopatia: uma Tríade, três Domínios e cinco Momentos. Arquivos Brasileiros de Cardiologia, 2005; 84(1): 44-48.

4. AVILA WS, et al. Gravidez em Portadoras de Cardiopatias Congênitas Complexas: Um Constante Desafio. Arq Bras Cardiol, 2019; 113(6): 1062-1069.

5. AVILA WS, et al. Posicionamento da Sociedade Brasileira de Cardiologia para Gravidez e Planejamento Familiar na Mulher Portadora de Cardiopatia-2020. Arquivos Brasileiros de Cardiologia, 2020; 114(5): 849-942.

6. BARBOSA PJB, et al. Diretrizes Brasileiras para Diagnóstico, Tratamento e Prevenção da Febre Reumática da Sociedade Brasileira de Cardiologia, da Sociedade Brasileira de Pediatria e da Sociedade Brasileira de Reumatologia. Arq Bras Cardiol, 2009; 93(3 Supl. 4): 1-18.

7. BORN D. 8. Cardiopatia congênita. Arquivos Brasileiros de Cardiologia, 2009; 93(6): 130-132.

8. CAVALCANTE MS, et al. Complicações maternas associadas à via de parto em gestantes cardiopatas em um hospital terciário de Fortaleza, CE. Rev Bras Ginecol Obstet, 2012; 34(3): 113-7.

9. COMITÉ COORDENADOR DA DIRETRIZ DE INSUFICIÊNCIA CARDÍACA, ROHDE LE, et al. Diretriz Brasileira de Insuficiência Cardíaca Crônica e Aguda. Arq Bras Cardiol, 2018; 111(3): 436-539.

10. DE MELO MOREIRA V. Cardiopatias Congênitas Complexas e Gravidez: Riscos Maternos e Fetais: Cardiopatias congênitas complexas e gravidez. Arquivos Brasileiros de Cardiologia, 2019; 113(6):1070-1071.

11. DRENTHEN W, et al. Investigators Z. Outcome of pregnancy in women with congenital heart disease: a literature review. J Am Coll Cardiol, 2007; 49: 2303-2311.

12. FELCZAK C, et al. Profile of cardiac pregnant women: high-risk. CogitareEnferm, 2018; (23)2: e49605.

13. GALVÃO ALC, GREZZANA GB. Doença cardíaca reumática crônica na gravidez. Revista da Sociedade de Cardiologia do Rio Grande do Sul, 2005; XIV(5):4p

14. GÓMEZ R, NAZAR C. Consideraciones generales de la embarazada con enfermedad cardiaca congénita y adquirida. Rev Chil Anest, 2013; 42: 77-87.

15. GUIMARÃES T, et al. Heart disease and pregnancy: State of the art. Rev Port Cardiol, 2019; 38: 373-383.

16. LAGE EM, BARBOSA AS. Cardiopatias e gravidez. FEMINA, 2012; 40(1): 43-50.

17. MENEGUINS, et al. Qualidade de vida de cardiopatas durante a gestação e após o parto. Acta Paulista de Enfermagem, 2016; 29(2): 232-238

18. MOREIRA WR, ANDRADE LC. Anestesia para gestante cardiopata. Rev Med Minas Gerais, 2009; 19(4) Supl 1: S21S62.

19. RAMLAKHAN KP, et al. Pregnancy and cardiovascular disease. Nature Reviews Cardiology, 2020: 1-14.

20. SAINTRAIN SV, et al. Fatores associados à morte materna em unidade de terapia intensiva. Rev Bras Ter Intensiva, 2016; 28(4): 397-404.

21. TEDOLDI CL, et al. Sociedade Brasileira de Cardiologia. Diretriz da Sociedade Brasileira de Cardiologia para Gravidez na Mulher Portadora de Cardiopatia. Arq Bras Cardiol, 2009; 93(6 supl.1): e110-e178. 